Research Paper

\title{
Significantly Increased Medical Expenditure on Breast Cancer Failing to Bring Down Its Mortality and Incidence Rate
}

\author{
Ming-Lin Ho ${ }^{1,2}$, Yung-Po Liaw ${ }^{3}$, Chien-Hsu Lai ${ }^{4}$, Yen-Yu Chen ${ }^{4}$,Horng-Der Tsai5, Ming-Chih Chou ${ }^{1,6}{ }^{凶}$, \\ Yi-Hsuan $\mathrm{Hsiao}^{7,5} \bowtie$ \\ 1. Institute of Medicine, Chung Shan Medical University, No. 110 Sec 1 Chien-Kuo N. Road, Taichung City 40201, Taiwan; \\ 2. Division of Pulmonary Medicine, Department of Internal Medicine, Changhua Christian Hospital, 135 Nanhsiao Street, Changhua, 500 \\ Taiwan; \\ 3. Department of Public Health and Institute of Public Health, Chung Shan Medical University, No. 110 Sec 1 Chien-Kuo N. Road, Tai- \\ chung City 40201, Taiwan; \\ 4. Department of Neurology, Changhua Christian Hospital, 135 Nanhsiao Street, Changhua, 500 Taiwan; \\ 5. Department of Obstetrics and Gynecology, Changhua Christian Hospital, 135 Nanhsiao Street, Changhua, 500 Taiwan; \\ 6. Division of Thoracic Surgery, Department of Surgery, Chung Shan Medical University Hospital, No. 110 Sec 1 Chien-Kuo N. Road, \\ Taichung City 40201, Taiwan; \\ 7. School of Medicine, Chung Shan Medical University, No. 110 Sec 1 Chien-Kuo N. Road, Taichung City 40201, Taiwan.
}

$\square$ Corresponding author: Yi-Hsuan Hsiao, M.D., PhD. Assistant Professor, School of Medicine, Chung Shan Medical University. Department of Obstetrics and Gynecology, Changhua Christian Hospital, 135 Nanhsiao Street, Changhua, 500 Taiwan. Phone: 886-4-7238595 Fax: 886-4-7228289 E-mail: 54315@cch.org.tw; Or Ming-Chih Chou, MD., PhD. Professor, Department of Surgery, Chung Shan Medical University, Taichung, Taiwan. Tel: 886-4-24730022 Fax: 886-4-24723229 E-mail: graduate@csmu.edu.tw.

(c) Ivyspring International Publisher. This is an open-access article distributed under the terms of the Creative Commons License (http://creativecommons.org/ licenses/by-nc-nd/3.0/). Reproduction is permitted for personal, noncommercial use, provided that the article is in whole, unmodified, and properly cited.

Received: 2013.03.08; Accepted: 2013.08.06; Published: 2013.08.10

\begin{abstract}
Background: The direct impact of medical expenses on breast cancer incidence and mortality rate has not been sufficiently addressed. The purpose of this study is to investigate the potential correlation between the incidence and mortality rate of breast cancer and the medical expenses in Taiwan.

Materials and Methods: Breast cancer cases were identified from the National Health Insurance Research Database (NHIRD) with corresponding to International Classification of Diseases, and the Ninth Revision (ICD-9) code 174, 1740-1749, I75, 1750 and 1759 from January 1999 to December 2006. Age-specific incidences were estimated by population data obtained from the Department of Statistics, Ministry of the Interior. Medical expenses, including outpatient and inpatient services, were also retrieved from the NHIRD.

Results: The incidence increased from 20.06 per 100,000 in 1999 to 30.34 per 100,000 in 2006; the total expenses increased from I,449,333,52I in 1999 to 4,350,400,592 Taiwan dollars in 2006. The age-standardized mortality rate for female breast cancer remained essentially unchanged, while the age-standardized incidence increased steadily (except 2002-2003). Among the top 20 coexisting ICD-9 codes for expenses, four are directly on cancers, while 16 are on other diseases or symptoms, which are not necessarily caused by breast cancer.

Conclusions: Significantly increased medical expenditure on breast cancer failed to bring down its mortality and incidence rate. The finding has implications for healthcare policy planners in proposing strategies for breast cancer control and allocating the resources.
\end{abstract}

Key words: incidence, medical expense, breast cancer, Taiwan. 


\section{Introduction}

More than one million women diagnosed with breast cancer every year are estimated $(1,2)$. In Taiwan, breast cancer has been the most common female cancer and the fourth cause of female cancer death since 2003 (3).

Breast cancer has been causing a health concern in women worldwide not only for its increasing incidence but also for the expense of treatment. Economic evaluation of breast cancer treatment is very valuable for providing information to healthcare policy planners, physicians, patients and others to assess the situation and allocate resources to cancer control strategies, understanding the quantity of breast cancer burden and the quality of treatment $(4,5)$. Published evaluations of costs for breast cancer treatment during the past varied substantially in perspective, methodology and patient populations $(6,7)$. To the best of our knowledge, the potential correlation between the medical expenses and breast cancer incidence and mortality rate has not been sufficiently addressed. Thus, our current study attempted to assess the impact of medical expenses on breast cancer incidence and mortality rate in Taiwan.

\section{Patients and Methods}

The National Health Insurance (NHI) service was implemented in Taiwan since 1995, and Cancer Registration was inaugurated since 1979. National Health Insurance (NHI) covers comprehensive medical care, including health prevention, clinical care, hospitalization, and social rehabilitation. Medical expenses, including outpatient and inpatient services, were also retrieved from the NHI database.

The National Health Insurance Research Database (NHIRD) is a large computerized database which is derived from the system by the Bureau of NHI and supported by the National Health Research Institute (NHRI). The NHIRD is supplied to the scientists in
Taiwan for aims of research. Before the database is released to each researcher, all the data in the NHIRD individual identification information, including patient ID, medical institutions, and care providers, are encrypted. Therefore, the approval of Institutional Review Board was waived for the study.

In Taiwan, the increased trend of breast cancer burden was noticed as a public health issue and the annual incidences of cancer have been published formally by Bureau of Health Promotion every year. In our study, the national cancer registry system (https://cris.bhp.doh.gov.tw/pagepub/Home.aspx) is also a data source.

Breast cancer cases were identified from the National Health Insurance Research Database (NHIRD) with corresponding to International Classification of Diseases, and the Ninth Revision (ICD-9) code 174, 1740-1749 (Malignant neoplasm of female breast), 175, 1750 and 1759 (Malignant neoplasm of male breast) from January 1999 to December 2006. Age -specific incidences were estimated by population data obtained from the Department of Statistics, Ministry of the Interior.

Calculations were carried out using SPSS 15.0 and Microsoft Excel 2003.

\section{Results}

Annual incidences of breast cancer were detailed in Table 1. During 1999-2006, the annual incidence ranged from 40.86 to 61.10 and from 0.20 to 0.42 per 100,000 for females and males, respectively. The combined incidences increase from 20.06 per 100,000 in 1999 to 30.34 per 100,000 in 2006.

Age-specific incidences (per 100,000/year) of female breast cancer were listed in Table 2 and Figure 1 . Peak incidence mostly fell on age $45-59$ years during 1999 to 2006. The increase in breast cancer incidence did not occur on women aged less than 35 .

Table I. Population, number of cases and incidence of breast cancer by year and gender.

\begin{tabular}{|c|c|c|c|c|c|c|c|c|c|}
\hline & \multicolumn{3}{|c|}{ total population (millions) } & \multicolumn{3}{|c|}{ breast cancer cases } & \multicolumn{3}{|c|}{ incidence rate (per $10^{5} /$ year) } \\
\hline & female & Male & Total & female & male & total & female & male & total \\
\hline 1999 & 10.78 & 11.31 & 22.09 & 4405 & 26 & 4431 & 40.86 & 0.23 & 20.06 \\
\hline 2000 & 10.88 & 11.39 & 22.28 & 4642 & 23 & 4665 & 42.65 & 0.2 & 20.94 \\
\hline 2001 & 10.96 & 11.44 & 22.41 & 5067 & 28 & 5095 & 46.22 & 0.24 & 22.74 \\
\hline 2002 & 11.04 & 11.49 & 22.52 & 5339 & 41 & 5380 & 48.38 & 0.36 & 23.89 \\
\hline 2003 & 11.04 & 11.49 & 22.6 & 5325 & 32 & 5357 & 48.25 & 0.28 & 23.7 \\
\hline 2004 & 11.15 & 11.54 & 22.69 & 6176 & 48 & 6224 & 55.4 & 0.42 & 27.43 \\
\hline 2005 & 11.21 & 11.56 & 22.77 & 6594 & 48 & 6642 & 58.83 & 0.42 & 29.17 \\
\hline 2006 & 11.28 & 11.59 & 22.88 & 6895 & 46 & 6941 & 61.10 & 0.40 & 30.34 \\
\hline
\end{tabular}


Table 2. Age-specific incidences (per 100,000/year) of female breast cancer by year.

\begin{tabular}{llllllllllllllll}
\hline $\begin{array}{l}\text { Age group } \\
\text { (year) }\end{array}$ & $\leqq 19$ & $20-24$ & $25-29$ & $30-34$ & $35-39$ & $40-44$ & $45-49$ & $50-54$ & $55-59$ & $60-64$ & $65-69$ & $70-74$ & $75-79$ & $80-84$ & $\geqq 85$ \\
\hline 1999 & 0.19 & 1.78 & 9.42 & 24.91 & 51.86 & 85.24 & 110.12 & 110.78 & 111.38 & 95.02 & 70.43 & 70.95 & 60.55 & 51.19 & 52.22 \\
2000 & 0.25 & 1.64 & 8.81 & 22.41 & 52.70 & 82.88 & 122.44 & 111.32 & 115.44 & 89.95 & 73.70 & 80.48 & 66.21 & 51.17 & 49.46 \\
2001 & 0.10 & 2.16 & 7.34 & 24.23 & 53.54 & 93.44 & 127.36 & 108.81 & 116.66 & 102.63 & 88.12 & 80.37 & 78.66 & 70.01 & 59.19 \\
2002 & 0.13 & 1.84 & 7.78 & 25.91 & 54.65 & 90.92 & 124.65 & 114.86 & 130.87 & 108.89 & 100.48 & 83.81 & 73.10 & 58.97 & 56.70 \\
2003 & 0.10 & 2.28 & 8.46 & 24.25 & 55.38 & 90.44 & 108.93 & 120.15 & 123.56 & 107.46 & 94.32 & 77.82 & 78.73 & 67.58 & 50.77 \\
2004 & 0.03 & 2.23 & 9.36 & 24.74 & 60.98 & 98.50 & 137.34 & 130.84 & 132.83 & 128.45 & 111.17 & 94.46 & 75.71 & 70.00 & 39.06 \\
2005 & 0.07 & 1.54 & 6.71 & 27.73 & 61.54 & 102.81 & 143.11 & 138.58 & 134.91 & 142.75 & 119.41 & 78.51 & 81.38 & 69.61 & 78.64 \\
2006 & 0.00 & 1.87 & 9.55 & 25.32 & 61.92 & 102.49 & 143.67 & 141.89 & 140.09 & 140.73 & 119.52 & 101.40 & 76.30 & 78.32 & 56.20 \\
\hline
\end{tabular}

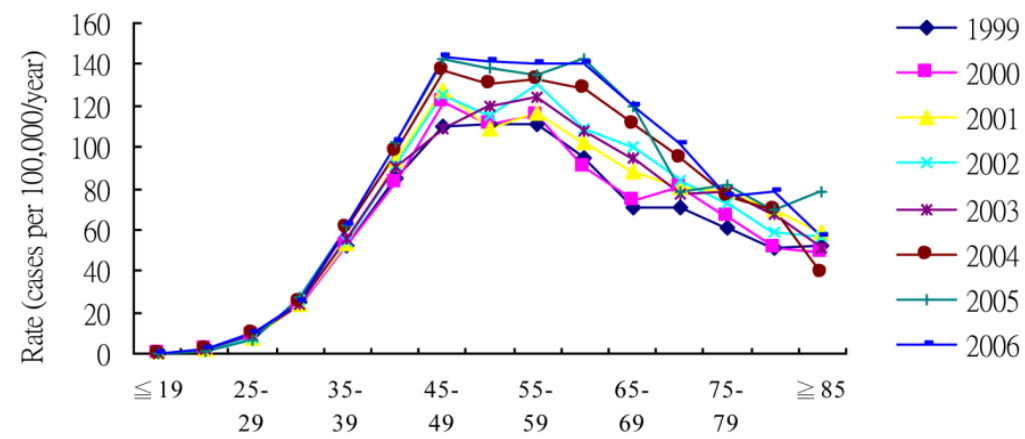

Age (years)

Fig I. Incidence rate by age groups in the period 1999-2006. Data sources: Taiwan cancer registration system.

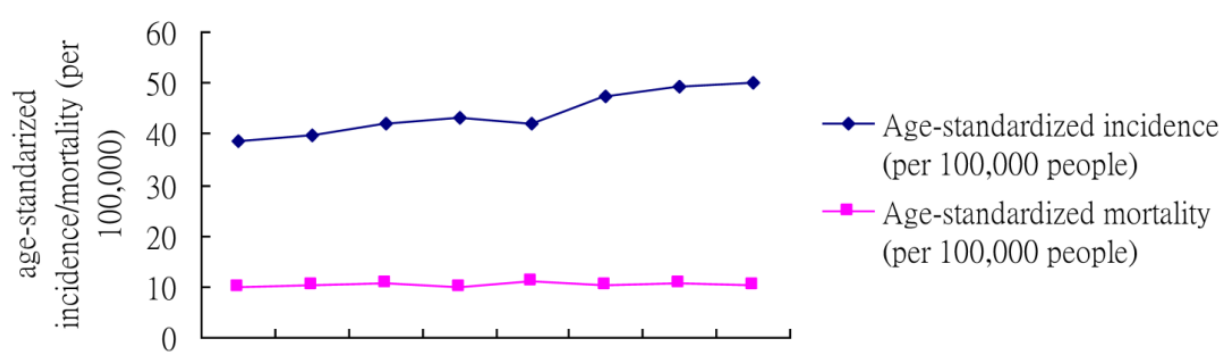

19992000200120022003200420052006

year

Fig 2. Age-standardized incidence (per 100,000 people) and Age-standardized mortality (per 100,000 people) of female breast cancer in Taiwan, 1999-2006. Data sources: Taiwan cancer registration system. Table I. Population, number of cases and incidence of breast cancer by year and gender.

Age-standardized incidence (per 100,000 people) and age-standardized mortality (per 100,000 people) of female breast cancer during 1999-2006 were shown in Figure 2. The age-standardized mortality rate for female breast cancer remains essentially unchanged, while the age-standardized incidence increased steadily (except 2002-2003).

Expenses of outpatient and inpatient services for breast cancer were detailed in Table 3. The total expenses increase from 1,449,333,521 in 1999 to 4,350,400,592 Taiwan dollars 2006.
The top 20 coexisting ICD-9 codes were listed in Table 4 . The most common coexisting ICD-9 code was V58.1 (Encounter for chemotherapy), followed by 401.9 (essential hypertension). Among the top 20 coexisting ICD-9 codes, four are directly on cancers, including [1] Encounter for chemotherapy, [2] secondary malignant neoplasm of bone and bone marrow, [3] Secondary and unspecified malignant neoplasm of lymph nodes of axilla and upper limb, and [4] secondary malignant neoplasm of lung. 16 of the top 20 are on other diseases or symptoms, which are not necessarily caused by breast cancer. 
Table 3. Number and total expenses of outpatient and inpatient services for breast cancer patients.

\begin{tabular}{llll}
\hline & Number of outpatient service & number of inpatient service & total expenses (TWD) \\
\hline 1999 & 233,460 & 15,834 & $1,449,333,521$ \\
2000 & 320,520 & 17,445 & $1,748,047,603$ \\
2001 & 349,191 & 18,285 & $1,884,945,689$ \\
2002 & 418,370 & 22,458 & $2,360,593,919$ \\
2003 & 449,415 & 28,472 & $2,858,184,684$ \\
2004 & 545,514 & 40,143 & $3,664,411,771$ \\
2005 & 624,332 & 42,083 & $4,111,497,959$ \\
2006 & 703,227 & 38,422 & $4,350,400,592$ \\
\hline
\end{tabular}

Table 4. The top 20 coexisting ICD-9 codes of breast cancer in Taiwan.

\begin{tabular}{|c|c|c|}
\hline ICD-9 code & Disease & frequency \\
\hline V581 & Encounter for chemotherapy & 149912 \\
\hline 4019 & Essential hypertension, unspecified & 112057 \\
\hline 4659 & Acute upper respiratory infections of unspecified site & 96892 \\
\hline 25000 & $\begin{array}{l}\text { Diabetes mellitus without mention of complication, Type II [non-insulin dependent type] [NIDDM type] } \\
\text { [adult-onset type] or unspecified type, not stated as uncontrolled }\end{array}$ & 81676 \\
\hline 78052 & Other insomnia & 75039 \\
\hline 1985 & Secondary malignant neoplasm of bone and bone marrow & 74155 \\
\hline 1963 & Secondary and unspecified malignant neoplasm of lymph nodes of axilla and upper limb & 47781 \\
\hline 6929 & Contact dermatitis and other eczema, unspecified cause & 43031 \\
\hline 5640 & Constipation & 34942 \\
\hline 1970 & Secondary malignant neoplasm of lung & 34344 \\
\hline 7291 & Myalgia and myositis, unspecified & 32371 \\
\hline 5369 & Unspecified functional disorder of stomach & 31304 \\
\hline 6272 & Menopausal or female climacteric states & 31010 \\
\hline 57140 & Chronic hepatitis, unspecified & 29792 \\
\hline 73300 & Osteoporosis, unspecified & 28474 \\
\hline 460 & Acute nasopharyngitis [common cold] & 28358 \\
\hline 78050 & Sleep disturbances, unspecified & 27920 \\
\hline 30000 & Anxiety state, unspecified & 26169 \\
\hline 7862 & Cough & 22272 \\
\hline 4011 & Benign essential hypertension & 19436 \\
\hline
\end{tabular}

\section{Discussion}

Our study showed that medical expenses of breast cancer treatment increased significantly and linearly from 1999 through 2006 in Taiwan (Table 3). However, the age-standardized mortality rate for female breast cancer remained essentially unchanged, while the age-standardized incidence increased steadily (except 2002-2003) (Figure 2). These facts indicate that significantly increased medical expenditure on breast cancer have failed to bring down its mortality and incidence rate.

The primary factors accounting for the significant increase of medical expenses on breast cancer appear to the followings: [1] A significant amount of the expenses are not directly related to cancer. In our study, among of the top 20 coexisting ICD-9 code (Table 4), four are directly on cancers. 16 of the top 20 are on other diseases or symptoms, which are not necessarily caused by breast cancer. [2] The applications of novel treatment regimens, including newly developed target therapy, hormone therapy and chemotherapy. Chemotherapy numbers were steadily increasing during the study period. Hormone therapy steeply increased since 2004. [3] A significant expansion of the breast cancer screening program. In Taiwan, the National Health Service breast cancer screening program was introduced in July, 2002 (3). After repeated review and improving, breast cancer screening services expand to female 45-69 years in 2009; and further, women aged 40-44 with a family history of breast cancer were covered since 2010. [4] 
Increasing incidences of more aggressive breast cancer types that require more comprehensive and costly treatment. A significantly higher incidence of axillary lymph node metastases for Taiwanese women with small breast cancer than for Western women was demonstrated (8). [5] Poor patients compliances for physicians' advice lead to disease advance and increase medical expenditure.

Our study showed that significantly increased medical expenditure on breast cancer failed to bring down its mortality and incidence rate in Taiwan. Furthermore, it might be a similar situation in other countries. The finding has implications for healthcare policy planners in proposing strategies for breast cancer control and allocating the resources.

Limitations of this study are stages of diseases and prognosis of the patients not clear due to database not support. The medical expenses may not reflect the variations for each stage of diseases. No connected mortality database for estimations of individual medical expense of treatment is also a limitation of our study.

\section{Acknowledgments}

This study is based in part on data from the National Health Insurance Research Database provided by the Bureau of National Health Insurance, Department of Health and managed by National Health Research Institutes. The interpretation and conclusions contained herein do not represent those of the Bureau of National Health Insurance, the Department of Health or National Health Research Institutes.

\section{Competing Interests}

The authors have declared that no competing interest exists.

\section{References}

1. Coughlin SS, Ekwueme DU. Breast cancer as a global health concern. Cancer Epidemiol. 2009; 33: 315-8.

2. Parkin DM, Bray F, Ferlay J, Pisani P. Global cancer statistics, 2002. CA Cancer J Clin. 2005; 55: 74-108.

3. Chang KJ, Kuo WH, Wang MY. The Epidemiology of Breast Cancer in Taiwan. J Chinese Oncol. 2008; 24: 85-93.

4. Imai H, Kuroi K, Ohsumi S, Ono M, Shimozuma K. Economic evaluation of the prevention and treatment of breast cancer--present status and open issues. Breast Cancer. 2007; 14: 81-7.

5. Parkin DM, Fernández LM. Use of statistics to assess the global burden of breast cancer. Breast J. 2006; 12 Suppl 1: S70-80.

6. Markman M, Luce R. Impact of the cost of cancer treatment: an internet-based survey. J Oncol Pract. 2010; 6: 69-73.

7. Yabroff KR, Lamont EB, Mariotto A, Warren JL, Topor M, Meekins A, Brown ML. Cost of care for elderly cancer patients in the United States. J Natl Cancer Inst. 2008; 100: 630-41.

8. Chao TC, Chen MF, Wang CS, Jan YY, Hwang TL, Chen SC. Small invasive breast carcinomas in Taiwanese women. Ann Surg Oncol. 2003; 10: 740-7. 